\title{
LA REVISTA DE HUMANIDADES DIGITALES: AÑO 1, VOLUMEN 1
}

\author{
Gimena del Rio Riande \\ IIBICRIT-CONICET \\ gdelrio@conicet.gov.ar
}

Las revistas científicas constituyen uno de los principales medios de comunicación para la comunidad académica. En ellas, los investigadores invierten su capital simbólico y se transforman en agentes de difusión -de trabajos propios y ajenos- y en fuentes de consumo de información. Scripta manent, verba volant, los resultados de la investigación científica necesitan de una periodicidad y de la escritura, así como de unas instituciones que otorguen garantías a las disciplinas académicas. $Y$ aunque hoy día, blogs y redes sociales hayan intervenido en la vida académica transformándose en vehículos ideales para una mejor difusión y visibilidad de las actividades de investigación, las publicaciones periódicas continúan siendo un elemento primordial en el circuito de producción y reproducción del saber con valor agregado.

Las Humanidades Digitales son un campo emergente y aún por definirse en diálogo con los contenidos curriculares actuales en las academias que utilizan el español como lengua de comunicación científica. No obstante, cuentan una larga historia que se remonta a mediados del siglo $X X$, cuando se cruzaron los intereses lingüísticos de un filósofo italiano y las posibilidades de explotación de una compañía norteamericana. La dupla Busa-IBM (Busa, 1980: 83-90) y, más tarde, la de Ellison y Remington-UNIVAC (Wisbey, 1965: 225), se cristalizaría para siempre en el congreso Computers for the Humanities?, de 1965, celebrado en la Universidad de Yale, y en la revista Computers and the Humanities, publicada tan solo un año más tarde, y hasta 2004, desde el Queens College of the City University of New York. Rescato del número inicial de este indispensable journal, las palabras inaugurales de su fundador y editor, Joseph Raben, de providencial claridad y actualidad, tanto con respecto al 
alcance de nuestras Humanidades Digitales, como a la (auto)justificación que atañe a toda publicación periódica de carácter científico:

The frequency with which new and specialized scholarly publications are appearing increases the need to justify each one (...) In our age of ephemeral vogues, in humanistic scholarship as in other activities, we must-as can never be over-repeatedstrive to recognize the permanent. In our age of intense specialization, we must vigorously pursue the vision of the human soul in all its varieties of expression. It is the hope that we can make our machines serve our higher aim that determines the order of climax in our title (Raben, 1966: 1).

Hoy, las Digital Humanities sostienen gran parte de su discurso (principalmente, en inglés) en una variada cantidad de revistas académicas como Digital Humanities Quarterly, Digital Studies/Le Champ Numérique, Journal of the Text Encoding Initiative, Journal of Digital Humanities o $\mathrm{DH}$ Commons, que pertenecen a una constelación textual en la que conviven con los Companions... (Schreibman et al., 2004; 2016), los Debates... (Gold et al., 2012; 2016) y una enorme cantidad de enciclopedias $\mathrm{DH}$. Por el contrario, en el caso de las Humanidades Digitales, más allá de los artículos y reseñas que circulan hoy publicados en revistas de carácter filológico, literario, bibliotecológico, o del ámbito de las Ciencias Sociales, contamos con dos volúmenes dedicados a ellas en Janus (2014) -recopilaciones de trabajos de congresos sobre el tema-, o con números especiales de publicaciones periódicas -no todas de acceso abierto y con diferente sistema de call for papers- como El Profesional de la Información (2014), el Anuario Americanista Europeo (2014), Ínsula (2015) o Virtualis (2016). $Y$ también con algunos monográficos en publicaciones científico-divulgativas, como ArtyHum (2015), y Actas de congreso en España y Argentina (Baraibar, 2014; Rio Riande et al., 2016).

Este primer número de la Revista de Humanidades Digitales (RHD) da cuenta de gran parte de las presentaciones del Segundo Congreso Internacional de la Sociedad Humanidades Digitales Hispánicas: Innovación, Globalización e Impacto, llevado a cabo en la Universidad Nacional de Educación a Distancia (UNED) de Madrid, del 5 al 7 de octubre de 2015. Creímos que, de algún modo, reunir estos veintidós trabajos en un volumen era una buena excusa para dar inicio a la revista, un plan de tan larga data como la organización del congreso y construido con la colaboración de colegas y amigos de la Universidad Autónoma de México (UNAM), Columbia University, el Consejo Nacional de Investigaciones Científicas y Técnicas de Argentina (CONICET) y la UNED.

La misión de la RHD es ser un espacio para que todas las voces que digan Humanidades Digitales puedan oírse desde lo abierto, desde la garantía y el rigor de la evaluación científica por pares y desde una comprensión extendida de las Humanidades Digitales (y de las Humanidades, al fin). Es ser un lugar de difusión y también de diálogo, y es entenderse como un proyecto que, al igual que muchos de los journals mencionados, busca 
crecer con la comunidad. Un proyecto que asume también responsabilidades que superan lo estrictamente académico y relacionado con las Humanidades Digitales: el acceso abierto, la defensa de del español como vehículo de comunicación científica sin desatender una reflexión crítica sobre las lenguas mayoritarias y la importancia de las lenguas minoritarias en el mundo, y la democratización del conocimiento más allá de toda frontera. Ese es su gran compromiso; el resto es, como siempre en las Humanidades, un work in progress.

\section{REFERENCIAS BIBLIOGRÁFICAS}

BUSA, R. (1980). "The Annals of Humanities Computing: The Index Thomisticus". Computers and the Humanities, 14.2, 83-90.

BARAIBAR, Á. (ed.) (2014). Visibilidad y divulgación de la investigación desde las Humanidades Digitales. Experiencias y proyectos. Pamplona: Servicio de Publicaciones de la Universidad de Navarra.

BURDICK, A, DRUCKER, J., LUNENFELD, P., PRESSNER, T., SCHNAPP, J. (2012). Digital_Humanities. Massachusetts: MIT Press.

GOLD, M. (2012, 2016). Debates in the Digital Humanities. Minnesota: University of Minnesota Press. Recuperado de http://dhdebates.gc.cuny.edu/ el 14/08/2017.

RABEN, J. (1966). "Prospect". Computers and the Humanities,1.1, 1-2.

RIO RIANDE, G. del (2915). La realidad de las Humanidades Digitales en España y América Latina. ArtyHum. Revista digital de Artes y Humanidades, monográfico 1, noviembre 2015, ISSN 2341-4898. Recuperado de https://www.artyhum.com/descargas/monograficos/MONOGRÁFICO HD.pdf el 14/08/2017.

RIO RIANDE, G. del, STRIKER, G. y CANTAMUTTO, L. (eds.) (2016). Las Humanidades Digitales desde Argentina. Tecnologías, Culturas, Saberes. Actas de las I Jornadas de Humanidades Digitales de la Asociación Argentina de Humanidades Digitales. Buenos Aires: Editorial de la Facultad de Filosofía y Letras de la Universidad de Buenos Aires. Recuperado de https://www.aacademica.org/jornadasaahd el 14/08/2017.

SCHREIBMAN, S., SIEMENS, R. y UNSWORTH, J. (2004). A Companion to Digital Humanities. Oxford: Blackwell. Recuperado de http://www.digitalhumanities.org/companion/ el 14/08/2017. (2016). A New Companion to Digital Humanities. Oxford: Wiley-Blackwell

WISBEY, R. (1965). "Computers and Lexicography". The Use of Computers in Anthropology, D. Hymes (ed.), 215-234. London-The Hague-Paris: De Gruyter Mouton. 